Original Article

\title{
Verification of the Correlation between Cognitive Function and Lower Limb Muscle Strength for the Community-dwelling Elderly
}

\author{
Hironori Ohsugi, PT, $\mathrm{PhD}^{1)^{*}}$, Shin Murata, PT, $\mathrm{PhD}^{1)}$, Atsuko Kubo, PT, $\mathrm{PhD}^{2}$, \\ Mizuki Hachiya, PT, MS ${ }^{2)}$, Aya Hirao, OT, $\mathrm{MS}^{2)}$, Kazuhiko Fujiwara, OT, $\mathrm{MS}^{2)}$, \\ Kenji KamiJou, OT, $\mathrm{PhD}^{2)}$ \\ 1) Faculty of Health Science, Kyoto Tachibana University: 34 Ohyakeyamada, Yamashina-ku, \\ Kyoto-city, Kyoto 607-8175, Japan \\ 2) Department of Rehabilitation Sciences, Nishikyusyu University, Japan
}

\begin{abstract}
Purpose] The purpose of this study was to evaluate the lower limb muscle strength of the community-dwelling elderly, with or without cognitive decline, using isometric knee extension strength (IKES) and the 30-second chair stand test (CS-30). [Subjects] A total of 306 community-dwelling elderly participated in this study. Assessment items were the CS-30, IKES, Mini-Mental State Examination (MMSE), and Trail-Making Test Part A (TMT-A). [Methods] Participants were divided into three groups according to their MMSE score: cognitive impairment (MMSE $\leq 24$ ), cognitive decline (MMSE 25 to 27), and normal (MMSE $\geq 28$ ). We compared IKES and CS-30 among the three groups. [Results] IKES was not significantly different among the three groups. However, the CS-30 was significantly different among the three groups. Upon further analysis the CS-30 score of each group, when adjusted for age and TMT-A, did not indicate a significant difference. [Conclusion] These results suggest that the lower limb muscle strength of the elderly does not differ with cognitive decline. Moreover, we suggest that when using the CS-30 score as an indicator of lower limb muscle strength attentional function should be taken into account.

Key words: Isometric knee extension strength, CS-30, Attentional function
\end{abstract}

(This article was submitted Apr. 14, 2014, and was accepted May 29, 2014)

\section{INTRODUCTION}

Populations are aging worldwide and patients with agerelated dementia are also increasing ${ }^{1}$. Many studies have investigated the physical function of patients with demen$\mathrm{tia}^{2)}$. These studies have found that patients with dementia have weaker lower limb muscles than healthy elderly people ${ }^{3)}$. However, some studies have shown no decrease in the lower limb muscle strength of patients with demen$\mathrm{tia}^{4)}$. Therefore, it is not clear whether the lower limb muscle strength of patients with dementia is less than that of healthy elderly people. The elderly with cognitive decline and dementia are at increased risk of falls compared to the cognitively normal elderly ${ }^{5}$. Lower limb muscle weakness has also been identified as a major risk factor contributing to falls by the elderly ${ }^{6}$ ). Therefore, the assessment of lower limb muscle strength is important among the elderly with cognitive decline and dementia.

Isometric knee extension strength (IKES) is often used to assess lower limb muscle strength in a research setting ${ }^{7)}$.

*Corresponding author. Hironori Ohsugi (E-mail: osugi@ tachibna-u.ac.jp)

(C2014 The Society of Physical Therapy Science. Published by IPEC Inc. This is an open-access article distributed under the terms of the Creative Commons Attribution Non-Commercial No Derivatives (by-ncnd) License $<$ http://creativecommons.org/licenses/by-nc-nd/3.0/> .
However, it is difficult to assess IKES in a clinical setting because special equipment is needed (e.g. a hand-held dynamometer [HHD]). On the other hand, the 30-second chair stand test (CS-30) uses only a chair and does not require a specific assessment setting, instrument, or technique. Therefore, the CS-30 is a popular method of assessing lower limb muscle strength in a clinical setting ${ }^{8)}$. The CS-30 was designed by Jones et al. ${ }^{9)}$, and its test-retest reliability and criterion-related and construct validity have been verified $^{9,10)}$. Nakatani et al. ${ }^{11)}$ adapted the CS-30 for Japanese people and validated the reliability and validity. In these studies, the CS-30 score was correlated with IKES $^{9-11)}$. Therefore, the CS-30 score is thought to reflect lower limb muscle strength. Recent studies ${ }^{12}$ ) have found that the CS30 score is affected by attentional function. This indicates that CS-30 scores are not only an indication of lower limb muscle strength but also of cognitive functions.

This study aimed to evaluate lower limb muscle strength among the community-dwelling elderly with or without cognitive decline, using IKES and the CS-30.

\section{SUBJECTS AND METHODS}

A total of 306 community-dwelling elderly (161 men and 145 women, mean age: 73.6 , SD: 6.6) participated in this study. All participants were healthy and without a medical history, such as stroke or rheumatoid arthritis, that would 
Table 1. The classification of participants and measurement data

\begin{tabular}{lccccc}
\hline & $\begin{array}{c}\text { Cognitive } \\
\text { impairment (a) }\end{array}$ & $\begin{array}{c}\text { Cognitive } \\
\text { decline (b) }\end{array}$ & Normal (c) & Post hoc test ${ }^{1}$ & Post hoc test $^{2}$ \\
\cline { 1 - 4 } $\mathrm{n}$ & 22 & 84 & 200 & & \\
\cline { 1 - 4 } Age, year & $76.0 \pm 5.8$ & $74.8 \pm 7.1$ & $72.8 \pm 6.4$ & & \\
IKES, kg/BW & $0.34 \pm 0.13$ & $0.39 \pm 0.10$ & $0.41 \pm 0.13$ & N.S. & N.S. \\
CS-30, times & $15.1 \pm 6.2$ & $19.4 \pm 5.4$ & $21.1 \pm 5.7$ & $\mathrm{a}<\mathrm{b}^{*}, \mathrm{a}<\mathrm{c}^{*}$ & \\
MMSE score & $22.6 \pm 1.5$ & $26.2 \pm 0.8$ & $29.2 \pm 0.8$ & & \\
TMT-A, sec & $167.0 \pm 64.8$ & $110.1 \pm 43.6$ & $94.6 \pm 27.6$ & & \\
\hline
\end{tabular}

Values are expressed as means \pm SD. N.S.: not significant, $*$ : $p<0.01$ IKES: Isometric knee extension strength; CS-30: 30-second chair stand test; MMSE: Mini-Mental State Examination; TMT-A: TrailMaking Test Part A. ${ }^{1}$ : Adjusted for age. ${ }^{2}$ : Adjusted for age and TMT-A.

have impaired their activities of daily living. Participants demonstrated independence in activities of daily living and participated in the study by walking or using public transportation to the research site. All participants volunteered for the study and gave their written informed consent to the use of all of the data. This study was approved by the Ethics Committee of Nishikyushu University (approval No. 216).

Assessment items were the CS-30, IKES, general cognitive function, and attentional function. The CS-30 was performed using the method standardized by Jones et $\mathrm{al}^{9}{ }^{9}$. Each participant was required to sit and then stand from a fixed chair as many times as possible in 30 seconds while keeping their arms folded across the chest. The number of times that the participant succeeded in standing upright in 30 seconds was recorded as the outcome variable.

IKES was assessed with an HHD ( $\mu$-Tas F1, ANIMA Co. Ltd., Tokyo, Japan $)^{7)}$. Participants were instructed to sit with their knees at 90 degrees of flexion. The HHD was attached by a strap to the leg just above the ankle. Participants were asked to push as hard as possible into the HHD and were given one trial per leg. The peak forces of both lower limbs were recorded and the mean was calculated. The value was then normalized to body weight because muscle strength is known to correlate with body weight ${ }^{13)}$.

General cognitive function was assessed using the MiniMental State Examination (MMSE). The MMSE comprises 11 major items which are scored, with a maximum possible total score of 30 points. Participants were divided into three groups according to their MMSE score ${ }^{14,15)}$ : cognitive impairment (CI; MMSE $\leq 24)$, cognitive decline (CD; MMSE 25 to 27), or normal (NO; MMSE $\geq 28$ ).

Attentional function was assessed with the Trail-Making Test Part A (TMT-A) ${ }^{16)}$. The TMT-A consists of numbers 1 to 25 scattered on a sheet of paper and participants must draw a line through consecutive numbers. The time required to complete the TMT-A task was measured for each participant.

The statistical analysis was performed using IBM SPSS Statistics 19 (SPSS, Inc., an IBM Company, Tokyo, Japan). The level of statistical significance was chosen as 0.05 . The correlations between the CS-30 and IKES in each group and all participants were assessed using the Pearson correlation coefficient. Analysis of covariance and the Bonferroni post hoc test were used to compare the CS-30 and IKES among groups using age as a covariate. Attentional function (TMT-A) is also known to influence the CS-30 $0^{12}$, and it was included in further analysis of covariance as a covariate.

\section{RESULTS}

The general cognitive function classification of the participants and measurement outcomes are listed in Table 1. The correlation between the CS-30 and IKES was significant in each group and for all participants (CI: $\mathrm{r}=0.51$; CD: $r=0.36$; NO: $r=0.44$; all participants: $r=0.45, p<$ $0.01)$. IKES was not significantly different among the three groups $(\mathrm{F}=2.79, \mathrm{p}=0.63)$. However, the $\mathrm{CS}-30$ was significantly different among the three groups $(F=8.96, p<0.01)$. The post hoc test indicated that the CS-30 score of CI group was significantly lower than those of the other two groups $(p<0.01)$. The results of further analysis, in which the CS30 scores of each group were adjusted for age and TMT-A, did not indicate a significant difference $(F=2.27, p=0.11)$.

\section{DISCUSSION}

We examined the lower limb muscle strength of the community-dwelling elderly by testing IKES and administering the CS-30. We divided participants into three groups based on cognitive function and compared the IKES and CS-30 results among the three groups. IKES was not significantly different, but the CS-30 showed a significant difference. However, further analysis of covariance using attentional function as the covariate revealed that there was no significant difference in the CS-30 scores among the three groups. These results suggest that the lower limb muscle strength of the elderly does not differ, irrespective of cognitive decline. Moreover, we suggest that when using the CS-30 score as an indicator of lower limb muscle strength attentional function should be taken into account.

The CS-30 may reflect lower limb muscle strength ${ }^{9-11)}$. The correlation between the CS-30 and IKES found in the present study was similar to a previous CS-30 study of Japanese people $(r=0.43)^{11)}$. It suggests that the CS-30 score reasonably indicates the lower limb muscle strength of community-dwelling elderly Japanese. A comparison of the CS-30 scores and IKES of the three groups revealed different results. IKES was not significantly different, but the CS30 score was significantly different among the three groups. 
The CS-30 score of the cognitive impairment group was lower than those of the other two groups. Further analysis of the CS-30, adjusting for attentional function, revealed that there was no significant difference among the three groups. Therefore, the results of this study suggest that lower limb muscle strength does not differ among the elderly irrespective of cognitive decline. These findings contrast with the findings of another study ${ }^{17}$, which found that people with cognitive impairment have impaired motor function and lower limb function. However, Eggermont et al. ${ }^{4)}$ did not find a significant difference in lower limb muscle strength among no cognitive impairment, mild cognitive impairment, and Alzheimer disease groups. Therefore, the results of our study support the results of Eggermont et $\mathrm{al}^{4}$ ). The basis of the association between muscle strength and cognitive decline is still unknown ${ }^{18)}$. In this study, we intended to show that community-dwelling elderly with poor cognitive function could maintain independence in activities of daily living. Their physical function might not differ. Consequently, we suggest that the lower limb muscle strength of the cognitive impairment group was maintained despite their lower cognitive function.

It should be noted that the result of comparing the CS-30 score was different when attentional function was included as a covariate. The CS-30 score is thought to indicate lower limb muscle strength ${ }^{19}$ ). The current study showed a correlation between the CS-30 score and IKES. However, the correlation coefficient was not high. This suggests that the CS-30 score may be dependent on another variable. A previous study ${ }^{12)}$ verified that the CS-30 score is affected by age, muscle endurance, and attentional function. The CS30 is a convenient and simple method for assessing lower limb muscle strength in a clinical setting. The CS-30 requires each participant to complete a sit-to-stand cycle as many times as possible in 30 seconds. This suggests that sustained attentional function is required to repeat the task with effort over the 30 seconds. Therefore, it is possible that participants who have lower attentional function would score lower on the CS-30 despite there being no significant decline in their lower limb muscle strength. We advocate that the CS-30 score should be considered together with the effect of attentional function level of a subject.

This study had some limitations. First, participants in this study were community-dwelling elderly without a previous diagnosis of dementia. Therefore, it is uncertain whether lower limb muscle strength was not really different in each cognitive function group. Second, we did not assess each participant's level of physical activity, and therefore cannot explain why lower limb muscle strength was not different among the groups. Further research among the elderly may reveal a correlation between lower limb muscle strength and cognitive function. Third, we did not examine gender differences because there was a shortage of participants. Gender differences may be revealed by analyzing more participants including those with dementia.

\section{REFERENCES}

1) Batsch N, Mittelman M: World Alzheimer Report. London: Alzheimer's Disease International, 2012

2) Blankevoort CG, van Heuvelen MJ, Boersma F, et al.: Review of effect of physical activity on strength, balance, mobility and ADL performance in elderly subjects with dementia. Dement Geriatr Cogn Disord, 2010, 30: 392-402. [Medline] [CrossRef]

3) Rosano C, Simonsick EM, Harris TB, et al.: Association between physical and cognitive function in healthy elderly: the health, aging and body composition study. Neuroepidemiology, 2005, 24: 8-14. [Medline] [CrossRef]

4) Eggermont LH, Gavett BE, Volkers KM, et al.: Lower-extremity function in cognitively healthy aging, mild cognitive impairment, and Alzheimer's disease. Arch Phys Med Rehabil, 2010, 91: 584-588. [Medline] [CrossRef]

5) van Doorn C, Gruber-Baldini AL, Zimmerman S, et al. Epidemiology of Dementia in Nursing Homes Research Group: Dementia as a risk factor for falls and fall injuries among nursing home residents. J Am Geriatr Soc, 2003, 51: 1213-1218. [Medline] [CrossRef]

6) Tinetti ME, Speechley M, Ginter SF: Risk factors for falls among elderly persons living in the community. N Engl J Med, 1988, 319: 1701-1707. [Medline] [CrossRef]

7) Katoh M, Yamasaki H: Comparison of reliability of isometric leg muscle strength measurements made using a hand-held dynamometer with and without a restraining belt. J Phys Ther Sci, 2009, 21: 37-42. [CrossRef]

8) Góes SM, Leite N, Shay BL, et al.: Functional capacity, muscle strength and falls in women with fibromyalgia. Clin Biomech (Bristol, Avon), 2012, 27: 578-583. [Medline] [CrossRef]

9) Jones CJ, Rikli RE, Beam WC: A 30-s chair-stand test as a measure of lower body strength in community-residing older adults. Res Q Exerc Sport, 1999, 70: 113-119. [Medline] [CrossRef]

10) Yamada T, Demura S: The relationship of force output characteristics during a sit-to-stand movement with lower limb muscle mass and knee joint extension in the elderly. Arch Gerontol Geriatr, 2010, 50: e46-e50. [Medline] [CrossRef]

11) Nakatani $T$, Nadamoto $M$, Mimura $K$, et al.: Validation of a 30-sec chairstand test for evaluating lower extremity muscle strength in Japanese elderly adults. Jpn Soc Phys Educ, 2002, 47: 451-461.

12) Ohsugi $H$, Murata $S$, Kubo A, et al.: [Attentional function relates with the score of a 30-s chair stand test]. Jpn J Health Promot, 2014, 16: 1-6 (in Japanese).

13) Bohannon RW: Reference values for extremity muscle strength obtained by hand-held dynamometry from adults aged 20 to 79 years. Arch Phys Med Rehabil, 1997, 78: 26-32. [Medline] [CrossRef]

14) Rait G, Morley M, Burns A, et al.: Screening for cognitive impairment in older African-Caribbeans. Psychol Med, 2000, 30: 957-963. [Medline] [CrossRef]

15) Singh-Manoux A, Akbaraly TN, Marmot M, et al.: Persistent depressive symptoms and cognitive function in late midlife: the Whitehall II study. J Clin Psychiatry, 2010, 71: 1379-1385. [Medline] [CrossRef]

16) Tombaugh TN: Trail Making Test A and B: normative data stratified by age and education. Arch Clin Neuropsychol, 2004, 19: 203-214. [Medline] [CrossRef]

17) Aggarwal NT, Wilson RS, Beck TL, et al.: Motor dysfunction in mild cognitive impairment and the risk of incident Alzheimer disease. Arch Neurol, 2006, 63: 1763-1769. [Medline] [CrossRef]

18) Boyle PA, Buchman AS, Wilson RS, et al.: Association of muscle strength with the risk of Alzheimer disease and the rate of cognitive decline in community-dwelling older persons. Arch Neurol, 2009, 66: 1339-1344. [Medline] [CrossRef]

19) Macfarlane DJ, Chou KL, Cheng YH, et al.: Validity and normative data for thirty-second chair stand test in elderly community-dwelling Hong Kong Chinese. Am J Hum Biol, 2006, 18: 418-421. [Medline] [CrossRef] 